\title{
A rare case of trifid mandibular canal with bilateral retromolar foramina
}

\author{
Quang Do ${ }^{1}$, Daniel Shen ${ }^{1}$, Hiroe Ohyama ${ }^{2}$, R. Shane Tubbs ${ }^{3,4,5,6}$, Joe Iwanaga ${ }^{3,7,8}$ \\ ${ }^{1}$ Harvard School of Dental Medicine, Harvard University, Boston, MA, ${ }^{2}$ Department of Restorative Dentistry and Biomaterials Sciences, Harvard \\ School of Dental Medicine, Boston MA, ${ }^{3}$ Department of Neurosurgery, Tulane Center for Clinical Neurosciences, Tulane University School of \\ Medicine, New Orleans, LA, ${ }^{4}$ Department of Anatomical Sciences, St. George's University, St. George’s, Grenada, ${ }^{5}$ Department of Structural \& Cellular \\ Biology, Tulane University School of Medicine, New Orleans, LA, ${ }^{6}$ Department of Neurosurgery and Ochsner Neuroscience Institute, Ochsner Health \\ System, New Orleans, LA, USA, ${ }^{7}$ Dental and Oral Medical Center, Kurume University School of Medicine, Kurume, Fukuoka, ${ }^{8}$ Division of Gross and \\ Clinical Anatomy, Department of Anatomy, Kurume University School of Medicine, Kurume, Fukuoka, Japan
}

\begin{abstract}
There are many reported anatomical variations of the mandibular canal. Consequently, there is great variation in the retromolar area, such as the quantity, size, and location of the retromolar foramen (RMF), the bony entrance of the retromolar canal (RMC). These variations allow for different accessory innervations to the mandibular molars and their adjacent buccal tissue because the RMC contains neurovascular bundles. Consideration of these anatomical variations is crucial for avoiding complications in anesthesia, implant placement, and surgery. However, the rarer canal types are often only imaged by computed tomography $(\mathrm{CT})$ or cone beam computed tomography (CBCT). We present a rare case with bilateral RMF and a unilateral trifid mandibular canal in a cadaver.
\end{abstract}

Key words: Mandible, Oral surgery, Anatomy, Anatomic variation, Cadaver

Received June 18, 2020; Revised July 11, 2020; Accepted July 13, 2020

\section{Introduction}

The mandibular foramen, the entrance of the mandibular canal, is located on the medial aspect of the mandibular ramus [1]. The mandibular canal contains the inferior alveolar neurovascular bundle that carries sensation and supplies blood to the mandible and mandibular teeth. While the mandibular canal typically has one singular trunk, there are reported anatomical variations of bifid and trifid mandibular canals. Each section of the mandibular canal may contain its own neurovascular bundle. Bifid and trifid mandibular canals can be classified into five main types according to the classification of Naitoh et al. [2] and expanded on by Rash-

\footnotetext{
Corresponding author:

Joe Iwanaga (iD

Department of Neurosurgery, Tulane Center for Clinical Neurosciences, Tulane University School of Medicine, New Orleans, LA 70112, USA E-mail: iwanagajoeca@gmail.com
}

suren et al. [3] depending on their course through the mandible.

One of the more common variation of bifid mandibular canal is the type 1 bifid mandibular canal, also known as the retromolar canal (RMC). This canal branches off from the mandibular canal distal to the third molar, often opening into the retromolar fossa as the retromolar foramen (RMF). The RMC can be grouped into five categories according to its course and morphology [4]. The RMC has been found to contain small arteries, veins, and myelinated nerve fibers. These nerves are often contiguous with the inferior alveolar nerve, though there are several studies discussed in the review by Ngeow and Chai [5] that show that the RMC and RMF can arise from other sources, such as branches of the buccal nerve. Regardless of their source, the nerves in the RMC have been reported to innervate the temporalis tendon, buccinator muscle, the most posterior region of the alveolar process, and the mandibular third molar tooth [6]. Some studies have also found that nerves in the RMC can inner- 
vate the gingiva of the mandibular molars, premolars, and mucosa of the retromolar region [4]. As a result, the locations of the RMF and the RMC have been noted by several studies to be an important consideration for surgical procedures such as third molar extraction, implant placement, and mandibular osteotomy in order to avoid complications. The RMC should also be considered in cases of inadequate anesthesia of mandibular molars [7]. A review of risk assessment of major anatomical variations in the mandible by Iwanaga et al. [8] suggested that the size of the RMF be considered since there may be an increased risk of neurosensory disturbance of the lower molar's buccal gingiva if the diameter of the RMF is $>2.3 \mathrm{~mm}$.

The RMC should not be confused with the accessory mandibular foramen (AMF), which is an anatomical variation often found superior to the mandibular foramen on the medial surface of the ramus. The AMF varies in sizes and can also be inferior, anterior, or posterior to the mandibular foramen. The AMF has been found to contain tributaries of the inferior alveolar vein, maxillary vein, and maxillary artery. It may also include a branch of the inferior alveolar nerve and artery if the AMF is superior to the mandibular canal. As a result, it should be located prior to osteotomy of the mandible to prevent excessive bleeding [9].

We present here a case of a trifid mandibular canal on the left and bilateral RMF, with a double RMF on the right side of the mandible.

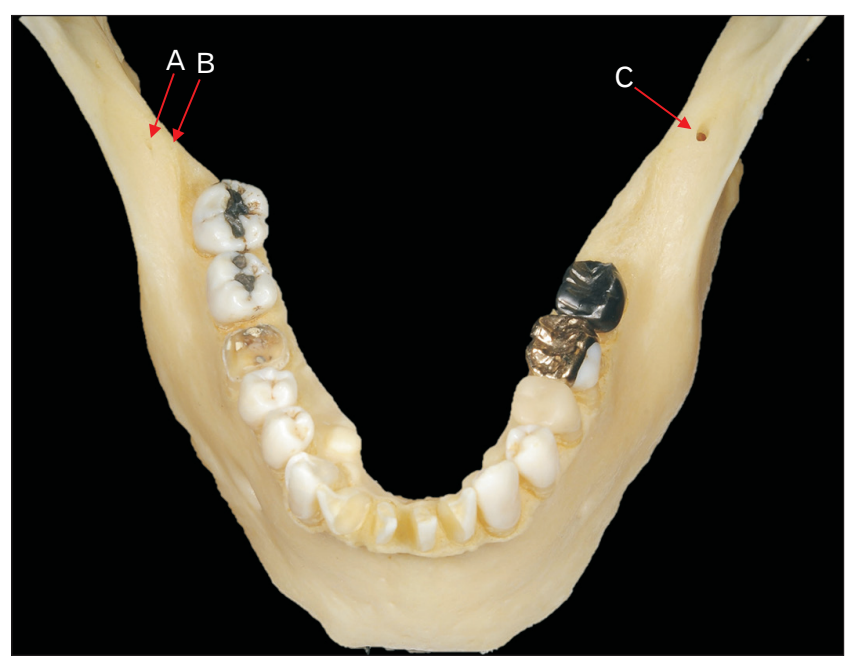

Fig. 1. A dry mandible demonstrating double retromolar foramina on the right $(\mathrm{A}, \mathrm{B})$ and a retromolar foramen on the left $(\mathrm{C})$. The three retromolar foramina are labeled.

\section{Case Report}

The mandible was resected from a 90-year-old at death male Caucasian cadaver. Bilateral RMF, single RMF on the left and double RMF on the right were noted (Fig. 1). The RMF were all observed in the retromolar trigone, between the internal and external oblique lines. An anatomical variation of the mandibular canal, a trifid canal, was also noted on the left side of mandible. The left mandibular canal after its entrance trifurcated into superior, middle, and inferior canals. The superior one was continuous with the left RMF (Fig. 2). The diameter of the RMF was measured using microcalipers (Mitutoyo, Kanagawa, Japan). The right RMF were labeled $\mathrm{A}$ and $\mathrm{B}$, with diameters of $0.93 \mathrm{~mm}$ and 0.56 $\mathrm{mm}$, respectively. The left $\mathrm{RMF}$ was labeled $\mathrm{C}$ with a diameter of $2.02 \mathrm{~mm}$. These variations were all observed under the surgical microscope with $\times 5, \times 10, \times 20$ magnified views (AmScope, Irvine, CA, USA) (Fig. 3).

The right mandibular canal was single while the left mandibular canal was trifid. No other variations, such as accessory mandibular foramina or accessory mental foramina were noted on this mandible.

\section{Discussion}

A trifid mandibular canal is a rare anatomical variation. According to Rashsuren et al. [3], bifid and trifid mandibular canals were found in $22.6 \%$ of 500 Korean patients via conebeam computed tomography (CBCT). The trifid canal type was found in only $5.8 \%$ of these multi-trunk canals [3].

In this case, there was a mandibular trifid canal but only one left RMF with a diameter of $2.02 \mathrm{~mm}$. It might be a type $5 \mathrm{~B}$ canal according to the classification system by Rashsuren

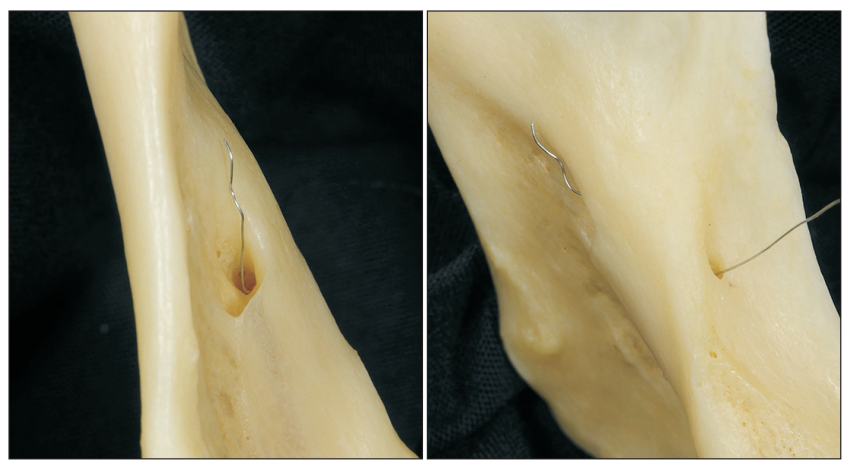

Fig. 2. Continuation of the retromolar foramen and superior canal on the left shown with a metal wire. 


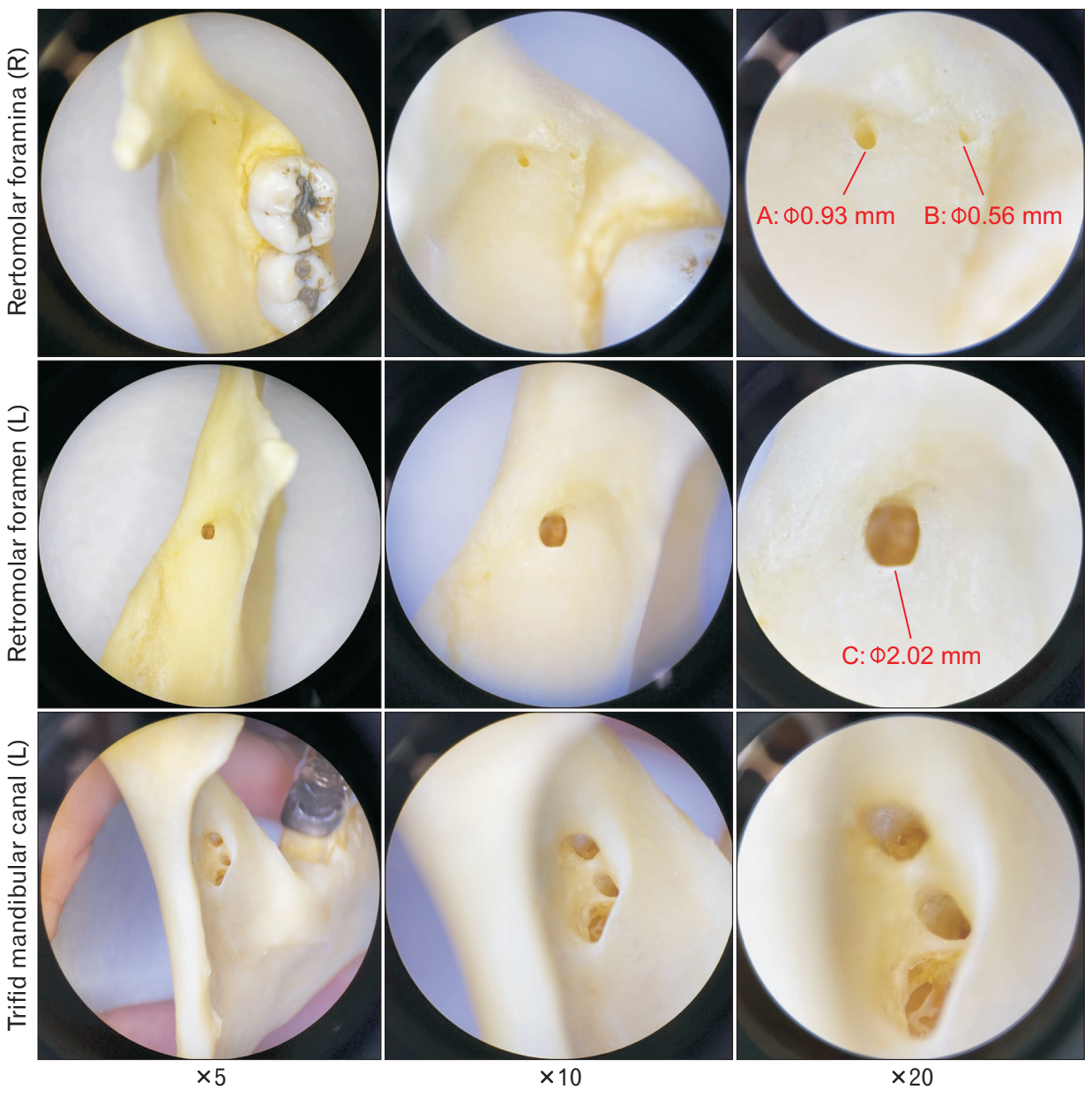

Fig. 3. A closer look at the retromolar foramina under the surgical microscope with $\times 5, \times 10, \times 20$ magnified view documenting their diameter. Note the trifid mandibular canal is observed from a posterior view. et al. [3], which is a trifid canal that has one main canal and two accessory canals (one RMC and one dental canal). The prevalence of RMF has been found to range from 12\% to $75 \%$ $[10,11]$. The wide reported range might be due to the method used to detect RMF. The diameter of the foramen is between $0.3 \mathrm{~mm}$ [12] and $2.29 \mathrm{~mm}$ [13]. Regardless of origin, the RMF may contain nerves that innervate the temporalis and buccinator muscles. Due to its location in this case, the left RMF may also innervate the posterior portion of the mandible, third molar teeth, gingiva of the mandibular molars and premolars, and mucosa of the retromolar pad. Histological study by Kim et al. [11] revealed that the RMC contained both artery and nerve. Due to such variations, failure of the standard inferior alveolar nerve (IAN) blockade may be due to inadequate anesthesia penetration or insertion so that all branches of the nerves that pass through the RMF and RMC are not reached. On the right side of the mandible, there was a rare double RMF, despite only one trunk in the mandibular canal. The presence of a double RMF has not been reported often in the literature [14].

Although small RMF might be overlooked during surgery, a large foramen such as the one found in the present case $(>2 \mathrm{~mm}$ ) might contain large neurovascular bundles and injury of such a large RMF during surgery might lead to neurosensory disorders or hemorrhage [13].

In conclusion, the anatomical variations shown in the present case may increase the risk of damage to such structures during mandibular surgeries and potentially complicate delivery of anesthesia. Further research is needed on the potential complications associated with a trifid mandibular canal.

\section{ORCID}

Quang Do: https://orcid.org/0000-0002-2765-937X

Daniel Shen: https://orcid.org/0000-0002-1990-9308

Hiroe Ohyama: https://orcid.org/0000-0002-3599-1671

R. Shane Tubbs: https://orcid.org/0000-0003-1317-1047 
Joe Iwanaga: https://orcid.org/0000-0002-8502-7952

\section{Author Contributions}

Conceptualization: HO, JI. Data acquisition: RST, JI. Data analysis or interpretation: QD, DS. Drafting of the manuscript: QD, DS. Critical revision of the manuscript: HO, RST, JI. Approval of the final version of the manuscript: all authors.

\section{Conflicts of Interest}

No potential conflict of interest relevant to this article was reported.

\section{Acknowledgements}

The authors wish to thank the individuals who donated their bodies for the advancement of education and research.

\section{References}

1. Iwanaga J, Watanabe K, Saga T, Tubbs RS, Tanaka K, Kikuta S, Tabira Y, Fisahn C, Kamura Y, Kusukawa J, Yamaki KI. A novel method for observation of the mandibular foramen: application to a better understanding of dental anatomy. Anat Rec (Hoboken) 2017;300:1875-80.

2. Naitoh M, Hiraiwa Y, Aimiya H, Ariji E. Observation of bifid mandibular canal using cone-beam computerized tomography. Int J Oral Maxillofac Implants 2009;24:155-9.

3. Rashsuren O, Choi JW, Han WJ, Kim EK. Assessment of bifid and trifid mandibular canals using cone-beam computed tomography. Imaging Sci Dent 2014;44:229-36.

4. von Arx T, Hänni A, Sendi P, Buser D, Bornstein MM. Radiographic study of the mandibular retromolar canal: an anatomic structure with clinical importance. J Endod 2011;37:1630-5.

5. Ngeow WC, Chai WL. The clinical significance of the retromolar canal and foramen in dentistry. Clin Anat 2020 Feb 4 [Epub]. http://dx.doi.org/10.1002/ca.23577.

6. Schejtman R, Devoto FC, Arias NH. The origin and distribution of the elements of the human mandibular retromolar canal. Arch Oral Biol 1967;12:1261-8.

7. Kodera H, Hashimoto I. [A case of mandibular retromolar canal: elements of nerves and arteries in this canal]. Kaibogaku Zasshi 1995;70:23-30. Japanese.

8. Iwanaga J, Kikuta S, Tanaka T, Kamura Y, Tubbs RS. Review of risk assessment of major anatomical variations in clinical dentistry: accessory foramina of the mandible. Clin Anat 2019;32:672-7.

9. Iwanaga J, Kikuta S, Ibaragi S, Watanabe K, Kusukawa J, Tubbs RS. Clinical anatomy of the accessory mandibular foramen: application to mandibular ramus osteotomy. Surg Radiol Anat 2020;42:41-7.

10. Motamedi MH, Gharedaghi J, Mehralizadeh S, Navi F, Badkoobeh A, Valaei N, Azizi T. Anthropomorphic assessment of the retromolar foramen and retromolar nerve: anomaly or variation of normal anatomy? Int J Oral Maxillofac Surg 2016;45:241-4.

11. Kim HJ, Kang H, Seo YS, Kim DK, Yu SK. Anatomic evaluation of the retromolar canal by histologic and radiologic analyses. Arch Oral Biol 2017;81:192-7.

12. Ogawa A, Fukuta Y, Nakasato H, Nakasato S. Evaluation by dental cone-beam computed tomography of the incidence and sites of branches of the inferior dental canal that supply mandibular third molars. Br J Oral Maxillofac Surg 2016;54:111620.

13. Kikuta S, Iwanaga J, Nakamura K, Hino K, Nakamura M, Kusukawa J. The retromolar canals and foramina: radiographic observation and application to oral surgery. Surg Radiol Anat 2018;40:647-52.

14. Kang JH, Lee KS, Oh MG, Choi HY, Lee SR, Oh SH, Choi YJ, Kim GT, Choi YS, Hwang EH. The incidence and configuration of the bifid mandibular canal in Koreans by using conebeam computed tomography. Imaging Sci Dent 2014;44:53-60. 